\title{
Cognitive Aging: A Common Decline of Episodic Recollection and Spatial Memory in Rats
}

\author{
R. Jonathan Robitsek, ${ }^{1}$ Norbert J. Fortin, ${ }^{1}$ Ming Teng Koh, ${ }^{2}$ Michela Gallagher, ${ }^{2}$ and Howard Eichenbaum ${ }^{1}$ \\ ${ }^{1}$ Center for Memory and Brain, Boston University, Boston, Massachusetts 02215, and ${ }^{2}$ Department of Psychological and Brain Sciences, Johns Hopkins \\ University, Baltimore, Maryland 21218
}

\begin{abstract}
In humans, recognition memory declines with aging, and this impairment is characterized by a selective loss in recollection of previously studied items contrasted with relative sparing of familiarity for items in the study list. Rodent models of cognitive aging have focused on water maze learning and have demonstrated an age-associated loss in spatial, but not cued memory. The current study examined odor recognition memory in young and aged rats and compared performance in recognition with that in water maze learning. In the recognition task, young rats used both recollection and familiarity. In contrast, the aged rats showed a selective loss of recollection and relative sparing of familiarity, similar to the effects of hippocampal damage. Furthermore, performance on the recall component, but not the familiarity component, of recognition was correlated with spatial memory and recollection was poorer in aged rats that were also impaired in spatial memory. These results extend the pattern of impairment in recollection and relative sparing of familiarity observed in human cognitive aging to rats, and suggest a common age-related impairment in both spatial learning and the recollective component of nonspatial recognition memory.
\end{abstract}

Key words: aging; hippocampus; recollection; familiarity; spatial memory; receiver operating characteristic

\section{Introduction}

In humans, aging is often associated with memory impairment, and in particular a decline in the capacity for recalling specific recent experiences, that is, in episodic memory (Craik and Byrd, 1982). Since the initial suggestion of age-associated memory impairment as a clinical diagnosis (Crook et al., 1986), much research has focused on understanding the changes in the brain systems that mediate the specific aspects of the observed deficits in aged humans (Albert, 1997; Buckner, 2004; Hedden and Gabrieli, 2004) and in animal models of cognitive aging (Gallagher and Rapp, 1997; Wilson et al., 2006). In humans, the pattern of deficits is highlighted by a disproportionate loss in the capacity for episodic recollection, similar to the pattern of deficits associated with a mild form of damage to the medial temporal lobes (Jennings and Jacoby, 1997; Bastin and Van der Linden, 2003; Quamme et al., 2004; Toth and Parks, 2006). In particular, signal detection analyses of recognition memory performance have revealed a striking dissociation in the receiver operating characteristic (ROC) function, revealing a pattern of impaired recollection and spared familiarity (Daselaar et al., 2006; Howard et al., 2006; Prull et al., 2006; Duverne et al., 2007). Correspondingly, the

Received April 29, 2008; revised June 17, 2008; accepted July 9, 2008

This work was supported by National Institutes of Health Grant AG09973. This manuscript was submitted in partial fulfillment of the requirements for the degree of Doctor of Philosophy in the Psychology Department at Boston University by R.J.R. We thank Lisa Guttentag and Shannon White for their excellent assistance in animal training and data collection.

Correspondence should be addressed to Howard Eichenbaum, Center for Memory and Brain, 2 Cummington Street, Boston, MA 02215.E-mail: hbe@bu.edu.

DOI:10.1523/JNEUROSCI.1893-08.2008

Copyright $\odot 2008$ Society for Neuroscience $\quad 0270-6474 / 08 / 288945-10 \$ 15.00 / 0$ deficit in recollection has been associated with decreased hippocampal activation (Daselaar et al., 2006).

Experimental studies on cognitive aging in rats have also revealed age-associated memory impairments that suggest a deterioration of hippocampal function (Rosenzweig and Barnes, 2003; Wilson et al., 2006). These analyses have focused on spatial learning and memory and highlight an impairment when performance requires the use of spatial cues, in contrast with intact memory when performance is guided by nonspatial stimuli (Rapp et al., 1987; Gallagher et al., 1993). Correspondingly, this impairment has been linked to multiple alterations of hippocampal anatomy and physiology, including decreases in synaptic densities, weakened synaptic plasticity, decreased cholinergic modulation, and a rigidity of spatial firing patterns of hippocampal neurons (Rosenzweig and Barnes, 2003; Wilson et al., 2006).

In humans and rats, not all aged individuals are impaired in memory relative to young controls, and the degree of impairment is typically associated with dysfunction of the medial temporal lobe. In humans, there is considerable individual variability in the degree of memory impairment and when aged study participants are separated into high- and low-performing groups, hippocampal activation in the high-performing elderly subjects is equivalent to that observed in young individuals (Duverne et al., 2007). Similarly, the degree of spatial memory impairment is highly variable in aged rats, and the severity of impairment in aged rats is related to the magnitude of change in multiple markers of hippocampal integrity (Gallagher and Rapp, 1997; Wilson et al., 2006).

Here, we sought to determine whether the age-associated impairment in recollection observed in humans extends to rats, and whether there is a relationship between performance on nonspa- 
tial recollection and in spatial memory. We used previously developed methods for characterizing the ROC of rats (Fortin et al. 2004) to show that, like young rats with hippocampal damage, aged rats have a selective impairment in recollection and relatively spared familiarity. We also exploited the variability in cognitive aging to relate the severity of the deficit in recollection to the impairment in spatial memory performance. These results suggest a common fundamental information processing failure that underlies both episodic recollection and spatial memory, both of which are critically dependent on the integrity of the hippocampus.

Parts of this paper have been presented previously (Robitsek et al., 2006).

\section{Materials and Methods \\ Subjects}

Subjects were 10 young (6-8 months) and 19 aged (22-24 months) male Long-Evans rats. The rats were initially tested for spatial learning ability in the Morris water maze at Johns Hopkins University and then shipped to Boston University for recognition memory testing. All testing occurred during the light phase of a $12 \mathrm{~h}$ light/dark cycle. Water was available ad libitum when rats were not undergoing testing. Before recognition testing, animals were allowed to acclimate to the laboratory for 1 week and then mildly food deprived to $85 \%$ of their ad libitum feeding weight for the remainder of the experiment. The experiment was conducted in accordance with guidelines set forth by the National Institutes of Health, and protocols were approved by the Johns Hopkins University and Boston University Charles River Campus Institutional Care and Use of Animals Committees.

\section{Water maze testing}

Spatial memory was assessed on the Morris water maze task using periodic probe trials to measure learning of the location of a submerged escape platform. Rats received three trials per day for 8 consecutive days using a $60 \mathrm{~s}$ intertrial interval and a $90 \mathrm{~s}$ cutoff to locate the platform on a trial. The location of the platform remained constant in one quadrant of the maze, and the starting position for each trial was varied among four equally spaced positions around the perimeter of the maze. Every sixth trial was a probe trial in which the platform was retracted for the first $30 \mathrm{~s}$, after which it was raised and made available for escape. Performance was measured by a spatial memory index calculated as the average proximity of the rat to the target platform location on probe trials two through four. Thus, low index values reflect stronger improvement in accurate search patterns during learning whereas high index values reflect inaccurate searching (Gallagher et al., 1993). In addition, on the day after spatial training, nonspatial guided performance was also assessed by presenting six cued training trials in which a visible platform $2 \mathrm{~cm}$ above the surface of the water was presented, and the location of the platform was varied randomly among the quadrants of the pool from trial to trial. On each trial, the rat was allowed $30 \mathrm{~s}$ to reach the platform and then allowed to remain there briefly before being returned to a holding cage for $5 \mathrm{~s}$ before the next trial.

\section{ROC analysis of recollection and familiarity in recognition memory}

Signal detection analysis of recognition memory in humans typically involves the initial presentation of a list of sample stimuli, then memory is assessed by asking subjects to distinguish re-presentations of the sample ("old") stimuli from additional ("new") stimuli. In addition, characterization of the ROC function in recognition requires testing across a range of biases that vary the criterion for classifying test stimuli as old or new; in experiments with humans subjects this is typically accomplished by asking subjects to rate their confidence in old and new decisions. The ROC function is then constituted as the probability of "hits," that is, correct identifications of old items, versus that for "false alarms," i.e., incorrect identifications of new items as old, at each bias or confidence level (see Fig. 4). The ROC function typically exhibits two major features that have been differentially associated with recollection and familiarity.
First, the ROC curve is asymmetrical such that the hit rate is elevated toward the left side of the curve, and this feature has been associated with high confidence recollection of items and their associations or source under more conservative bias levels. Second, the shape of the ROC function is typically curvilinear, and the degree of curvature, i.e., the "bowing" of the curve, reflects the strength of familiarity. The distinction between these components, and measurement of them in recollection and familiarity parameter estimates, is supported by a large literature (Yonelinas, 2002) (but see Wixted, 2007; Yonelinas and Parks, 2007). Among the most compelling evidence for these distinctions are studies showing that requiring subjects to rely on recollection of associations between studied items results in an ROC curve that is asymmetrical but linear, whereas requiring subjects to rely on familiarity for rapid recognition judgments results in an ROC curve that is curvilinear but symmetrical.

\section{ROC analysis of recognition memory in rats}

We have developed a version of this task for rats using common household odors as the stimuli and variations in the payoff ratio for correct responses to manipulate criterion, or bias for old and new responses (Fig. 1) (Fortin et al., 2004). Rats were trained to perform this task in a series of stages beginning with presentation of single sample stimuli after which they were required to distinguish a subsequent single test stimulus as old or new using a nonmatching to sample rule. Progressively longer lists of sample and test stimuli were then introduced, and finally the subjects' response biases were manipulated by altering the reward payoffs for correct old and new responses (Fig. 1).

Shaping. One week after food deprivation began, $125 \mathrm{ml}$ of Nalgene cups filled with $100 \mathrm{~g}$ of sand were baited with 10 buried and 10 halfburied Froot Loop pieces (Kellogg's). The cups were secured with Velcro on a Plexiglas base and left in the home cage in the vivarium. One hour later, the cups were checked, and rebaited for a second presentation. Over the course of the remaining week, the cereal rewards were transitioned toward being completely buried, and most rats readily approached the cup and obtained all rewards within a few minutes. Once rats would readily dig to obtain the cereal rewards, they were brought to the testing room and allowed to practice approaching the sand-filled cup to obtain single buried rewards. This procedure was repeated until all rats would approach the cup and dig quickly such that they completed 20 trials within $10 \mathrm{~min}$.

Training. Subsequently, rats were trained on the nonmatch to sample rule. Each stimulus cup was filled with $100 \mathrm{~g}$ of sand that was scented by mixing in $0.5-1 \mathrm{~g}$ of a common household spice and baited with one-half of a Froot Loop. The stimulus cup was presented at the front end of the home cage and a small empty cup was placed in the back of the cage that remained there for the duration of testing. At the outset of each trial, the rat was forced to the back of the cage using a Plexiglas barrier, a scented sample cup was placed in the front of the cage, the barrier was removed, the rat was freed to obtain reward, then it was again forced to the back of the cage. After a short memory delay $(\sim 5 \mathrm{~s})$, either the same odor (a "match") or a different odor ("nonmatch") was placed at the front of the cage, and the rat was allowed to approach it. Nonmatching odors were baited, such that the rat could receive one-half of a Froot Loop for digging in a nonmatching stimulus. Matching test stimuli were not baited, but if the rat refrained from digging in the test cup for three seconds, a whole Froot Loop was placed in the empty cup at the back of the cage and the animal was allowed to retrieve it. No reward was given for incorrect responses, i.e., not digging in test cups with a nonmatching odor or digging in test cups with a matching odor. Rats were tested on 10 trials per day, each consisting of a sample stimulus and a subsequent test. On each day, the odors were pseudorandomly selected from a pool of 40 odors and counterbalanced across days to ensure equal overall familiarity. Training on this stage continued until subjects performed $80 \%$ correct over $3 \mathrm{~d}$.

In the next stages of training, the number of sample odors presented sequentially before testing was increased to five and the test list was increased to 10 odors, then subsequently the sample list was increased to 10 odors and the test list was increased to 20. At each of these stages of training, a criterion of $80 \%$ correct over $3 \mathrm{~d}$ was required before progress- 
A

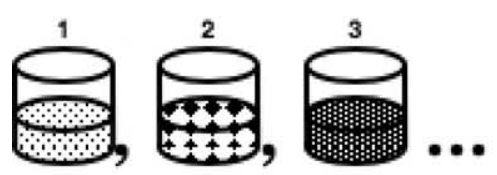

B
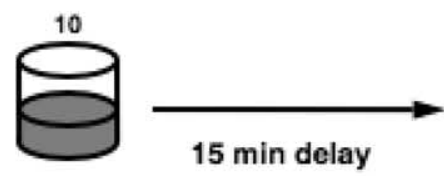

c

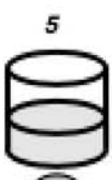

(O) Froot Loops

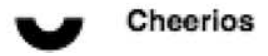

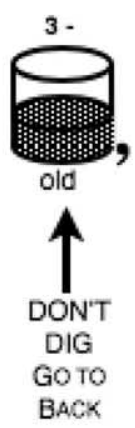

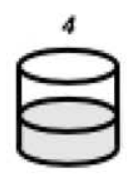

$\circlearrowleft$

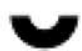

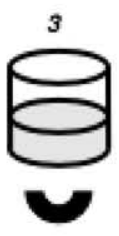

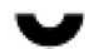

Test
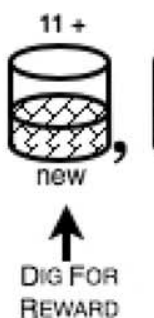

REWARD
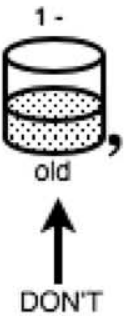

DON'T

DIG

GO TO

BACK
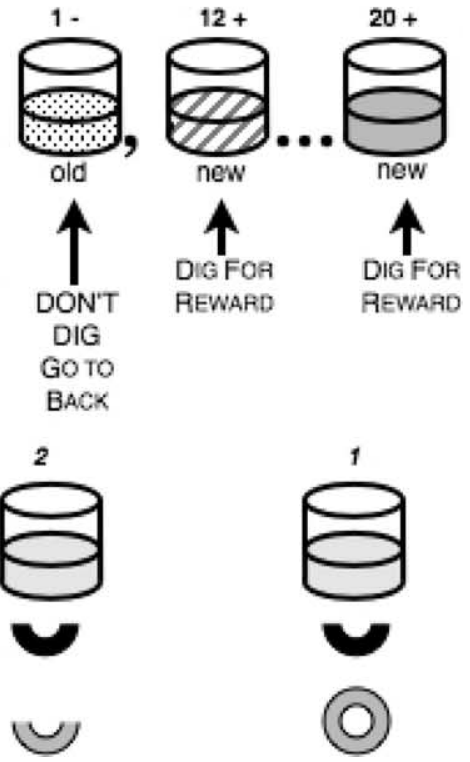

Figure 1. Odor-recognition task. $\boldsymbol{A}$, During the study phase, rats are presented with a sequence of 10 odors (represented by different patterns) mixed in sand baited with a buried reward. $\boldsymbol{B}$, After a 15 min delay, in the test phase, 10 new odors are singly presented in intermixed order with the 10 old odors. Rats can dig for reward in the new odors and must refrain from digging to obtain reward elsewhere if the odor is old. C, Five levels of response bias were generated by varying the desirability (Froot Loop vs Cheerio) and amount (0.5 or 1 piece) of reward.

ing to the next stage. Multiple lists were used across testing sessions, and sample odors were randomly intermixed with new odors during the test phase so that there was no reliable way for the rats to use the order of odor presentation during the sample phase to aid performance in the test phase. During training on the 10-odor phase, the delay between the sample and choice phase was gradually increased to $15 \mathrm{~min}$. When animals reached the performance criterion with 10 sample and 20 test odors, new reward payoff assignments were introduced. Five different response biases were generated by varying the amount of reward ( 0.5 or 1 piece of cereal) and by varying the desirability of the reward (Froot Loop vs Cheerio, half vs full piece) (Fig. 1).

In subsequent testing used to generate the ROC functions, the first four test odors of each session were used to inform the subject of the bias level for that session and were therefore excluded from data analysis. A behavioral response was scored as a hit when the rat correctly withheld digging on old test odors and as a false alarm when the rat incorrectly withheld digging on new test odors. The ratio of hits and false alarms over four testing sessions was calculated for each bias level for each rat, and averages of these values were used to construct the ROC functions for each subject group. In addition, the overall percentage of correct responses $[(p \mathrm{Hit}+(1-p \mathrm{FA})) / 2 \times 100]$ was calculated for each rat.

ROC functions and parameter estimates of recollection and familiarity components of the ROC function were derived from the observed hit and false alarm rates across the five bias levels using a least-squares method based on the dual-process signal-detection model (DPSD) (Yonelinas et al., 1998). Briefly, performance measured at five bias levels yield a system of 10 simultaneous equations that are combined to produce an ROC function that fits the raw hit and false alarm rate data by minimizing the sum of squared error between the predicted functions of the model and the observed data. The model posits that the probability of a hit (respond old|stimulus = old) is equal to the probability that an old item is recollected $(R)$, or that it is not recollected $(1-R)$ and the familiarity of the old item exceeds an internal response criterion set by the subject $\left(F_{\text {old }}\right): P$ $($ old $\mid$ old $)=R+(1-R)\left(F_{\text {old }}\right)$. The probability of a false alarm (respond old $\mid$ stimulus $=$ new $)$ is equal to the probability that the familiarity of the old item exceeds an internal response criterion $\left(F_{\text {new }}\right): P($ old $\mid$ new $)=$ (Fnew). Familiarity $\left(F_{\text {old }}\right.$ and $\left.F_{\text {new }}\right)$ is described as a signal detection process (Macmillan and Creelman, 2005), and the probability that old and new items will be identified as old based on familiarity is a function of the degree to which studied items are more familiar than new items
( $d$ '; the distance between the means of the old and new item distributions) and response criterion (the tendency to declare a given stimulus old or new). To facilitate comparisons between estimates of recollection, which is measured as a probability, and familiarity, which is measured as $d^{\prime}$, each rat's $d^{\prime}$ value was converted to the probability of a hit given a false alarm rate of 0.032 (Yonelinas, 2002).

Additional analyses calculated linear fits for $z$-transformed hit and false alarm rates, which provide supporting information about the shape of the ROC curve. The individual hit and false alarm rates at each bias were transformed into $z$-scores by taking the inverse of the standard cumulative normal distribution, assuming a mean of 0 and a SD of 1 . The slope of the $z$-transformed ROC (zROC) provides an index of the degree of asymmetry present in the recognition ROC, which, according to the DPSD model, is indicative of the degree of the contribution of recollection to recognition performance (Yonelinas et al., 1998). If the empirical ROC is symmetrical about the diagonal, the corresponding zROC will have a slope not significantly different from 1 , suggesting that recollection does not contribute significantly to item recognition performance. If the observed ROC is asymmetrical along the diagonal, appearing to be shifted up and to the left, the $\mathrm{zROC}$ slope will be $<1$ indicating that recollection does contribute to recognition performance. Linear regression analysis was performed on the individual and group $z$-transformed ROC data to determine whether the slopes deviated from 1 .

\section{Results}

\section{Spatial learning}

As has been shown previously (Gallagher et al., 1993), aged rats (22-24 months) had a significantly higher spatial memory index compared with young rats (6-8 months), indicative of impaired spatial memory $\left(257 \pm 11\right.$ vs $200 \pm 8 \mathrm{~cm}$, mean $\pm \mathrm{SEM} ; t_{(25)}=$ $4.23 ; p=0.0003$ ). As shown in Figure 2, some of the aged rats had scores within the range of young performance, whereas others performed outside the normative range (index score, $>240$ ) of the young subject population (Gallagher et al., 1993). The aged rats that performed outside the range of the young rats were designated as "spatial impaired" $(n=11)$, whereas those whose performance was within the range of young rats were designated "spatial unimpaired" $(n=8)$. 
Water Maze Performance

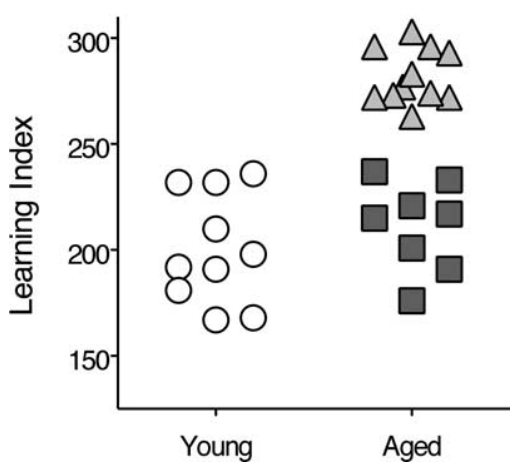

Figure 2. Water maze performance. Learning index scores reflect the average distance from the platform location in probe tests such that lower values represent a more accurate search for the escape platform. Aged rats that performed as well as young rats (circles) are designated spatial unimpaired (squares); aged rats that performed outside the normal range are designated spatial impaired (triangles).

\section{Overall recognition performance}

Overall performance in terms of the percent correct for all trials used to generate the ROC functions was not significantly different between aged and young rats $\left(F_{(1,27)}=1.2 ; p=0.3\right)$ (Fig. $3 A$ ), nor were there any significant differences in overall recognition memory performance between the groups of young, spatialunimpaired and spatial-impaired aged rats $\left(F_{(2,26)}=1.08 ; p=\right.$ 0.35 ) (Fig. 3B). Nevertheless, there was a significant linear relationship between water maze index scores and overall recognition memory performance $\left(\beta=-0.472 ; t_{(27)}=-2.78 ; p=0.01\right)$ (Fig. $3 C$ ), with water maze performance accounting for a significant proportion of the variance in overall performance on the recognition memory task $\left(r^{2}=0.22 ; t_{(27)}=-2.78 ; p=0.01\right)$.

Separate regressions for the subject groups indicated that water maze index scores and overall performance on recognition were correlated in young rats $\left(\beta=-0.78 ; r^{2}=0.61 ; t_{(8)}=\right.$ $-3.54 ; p=0.01)$, and the correlation approached significance in spatial-unimpaired aged rats $\left(\beta=-0.68 ; r^{2}=0.46 ; t_{(6)}=2.25\right.$; $p=0.06)$ but not in spatial-impaired aged animals $\left(\beta=-0.8 ; r^{2}\right.$ $\left.=0.006 ; t_{(9)}=0.23 ; p=0.83\right)$.

\section{ROC functions in recognition memory}

The signal detection analysis of recognition memory revealed that different processes support recognition performance in young and aged rats. The ROC function of young rats was significantly curvilinear according to a model-independent quadratic regression $\left(F_{\mathrm{QUAD}}(2,2)=24.8 ; p=0.04\right)$ (Fig. $4 A$ ), and to the DPSD model $\left(d^{\prime}=1.08 ; d^{\prime}\right.$ significantly $>0, t_{(9)}=8.28, p<$ $0.0001)$, suggesting that a familiarity process contributed to the ROC curve (Yonelinas et al., 1998). The ROC of young rats was also highly asymmetric (zROC slope $<1, t_{(9)}=-4.2, p=0.002$, with a positive Y-intercept; $r=0.32 ; R$ significantly $>0, t_{(9)}=$ $5.836, p=0.0002)$, indicating the presence of a recollection component as well (Yonelinas et al., 1998). The ROC function of aged rats also had a significant curvilinear component $\left(d^{\prime}=1.32 ; d^{\prime}\right.$ significantly $>0, t_{(18)}=10.35, p<0.0001 ; F_{\mathrm{QUAD}(2,2)}=34.2, p=$ 0.03 ) (Fig. $4 B$ ), yet was fully symmetrical and intersected the origin $(r=0)$, indicating performance based primarily on familiarity. The parameter estimates from the DPSD model further indicate qualitative distinctions in the mechanisms supporting performance by the two groups provided by the shape of the ROC functions. Familiarity estimates did not differ between the two groups $\left(F_{(1,27)}=0.65 ; p=0.43\right)$, but the recollection estimate for the aged group was significantly lower than that of the young group $\left(F_{(1,27)}=4.7 ; p=0.008\right)$ (Fig. $\left.4 C\right)$. These findings indicate the age-related deficit in recognition performance is attributable to a selective impairment in recollection.

Additional analyses were aimed at determining the relationship between the patterns of performance on the recognition task with that on the water maze task by comparing the performance of the young group with aged groups designated spatial unimpaired and spatial impaired. As in young rats, the ROC function of spatial-unimpaired aged rats had a significant curvilinear component $\left(d^{\prime}=1.2 ; d^{\prime}\right.$ significantly $>0, t_{(7)} 8.83, p<0.0001 ; F_{\mathrm{QUAD}}$ $(2,2)=147.1 ; p=0.007)$, as well as an asymmetric component reflected by a positive Y-intercept $(r=0.16 ; R$ significantly $>0$, $\left.t_{(7)}=4.06, p=0.0048\right)$, indicating performance based on both recollection and familiarity (Fig. $4 D$ ). In sharp contrast, the ROC of the spatial-impaired group was fully symmetrical and intersected the origin $\left(r=0 ; R\right.$ not significantly $>0, t_{(10)}=1.779, p=$ $0.11)$, but was curvilinear $\left(d^{\prime}=1.3 ; d^{\prime}\right.$ significantly $>0, t_{(10)}=$ $\left.6.9, p<0.0001 ; F_{\mathrm{QUAD}}(2,2)=39.45 ; p=0.007\right)$, suggesting that recognition was supported solely by familiarity (Fig. $4 E$ ). When the contribution of recollection was algebraically subtracted from the ROC function of young rats and spatial-unimpaired aged rats, the respective ROC curves became superimposed on that of the ROC function of the spatial-impaired rats, providing additional evidence that the differences in recognition performance between the groups are mostly accounted by the differences in their capacity for recollection.

Furthermore, recollection estimates significantly differed between groups $\left(F_{(2,26)}=9.25 ; p=0.0009\right)$ (Fig. $\left.4 F\right)$. Post hoc analyses indicated that there was no difference between young and spatial-unimpaired aged rats in estimates of recollection $\left(t_{(16)}=1.08 ; p=0.33\right)$, but the recollection estimate of spatialimpaired rats was decreased relative to that of young rats $\left(t_{(19)}=\right.$ $4.15 ; p=0.0005)$ and spatial-unimpaired aged rats $\left(t_{(17)}=2.87\right.$; $p=0.01)$. There were no group differences in familiarity estimates (derived from $d^{\prime}$ measure; $F_{(2,26)}=0.355 ; p=0.23$ ), although a trend toward increased familiarity in both the spatialunimpaired and spatial-impaired aged groups relative to the young rats was notable. This pattern of results suggests that recollection was absent in spatial-impaired rats but the contribution of familiarity to performance was similar among all three groups, as observed in ROC studies on rats with hippocampal lesions and aged humans (Fortin et al., 2004; Prull et al., 2006).

As can be seen in Figure $3 C$, there is a large amount of variability in the recognition performance of spatial-impaired aged animals, such that almost half of the animals are able to attain relatively high levels of performance, whereas the remainder of the group performs rather poorly. Further separating the spatialimpaired aged animals into subgroups who performed above and below the regression line yielded an SI-high group (subgroup that had higher overall recognition scores) with a mean recognition performance of $75.8 \pm 1.6 \%$ and an SI-low group (subgroup that had lower overall recognition scores) with a mean performance of $64.1 \pm 1.3 \%$, respectively. Bonferroni-corrected multiple comparisons $\left(\alpha=0.05 ; p_{\text {crit }}<0.008\right)\left(p_{\text {crit }}\right.$ is critical value that a $p$ value must be below to be significant when making multiple comparisons) revealed that the SI-low animals performed significantly worse than young rats $\left(t_{(14)}=4.56 ; p=0.0004\right)$ and spatial-unimpaired aged rats $\left(t_{(12)}=3.41 ; p=0.005\right)$, as well as SI-high rats $\left(t_{(9)}=4.19 ; p=0.002\right)$. The SI-high rats did not perform differently than young or spatial-unimpaired aged rats (both $p$ values $>0.05$ ). 


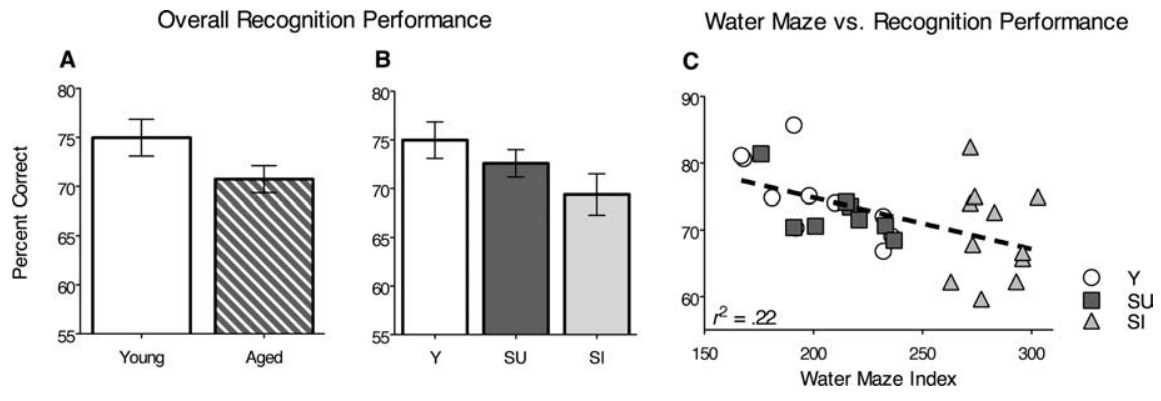

Figure 3. Overall recognition memory performance measured as the percentage correct on all tests ( \pm SEM). $\boldsymbol{A}$, Overall performance of young and all aged rats on the recognition memory task. $\boldsymbol{B}$, Performance of young rats, aged rats that were unimpaired in spatial memory (SU), and aged rats that were impaired in spatial memory (SI). C, Correlation between overall recognition memory scores and spatial memory performance.

The ROC curves of both SI-high $\left(r=0 ; d^{\prime}=1.7\right)$ (Fig. 4G) and SI-low $\left(r=0 ; d^{\prime}=0.94\right)$ (Fig. $\left.4 H\right)$ rats were fully symmetrical and intersected the origin, reflecting a complete loss of recollection in both groups. Because of the reduced number of subjects in those subgroups, the quadratic regression used previously was not sensitive enough to show that those ROCs were significantly curvilinear (SI-high, $F_{\mathrm{QUAD}}(2,2)=2.17, p=0.32$; SI-low, $F_{\mathrm{QUAD}}$ $(2,2)=11.5, p=0.08)$. However, as for the other ROCs, the group $d^{\prime}$ values were significantly $>0$ (SI-high, $t_{(4)}=7.30, p=0.002$; SI-low, $\left.t_{(5)}=6.82, p=0.001\right)$, confirming the observation that the ROCs are indeed curvilinear.

Estimates of recollection were correspondingly low in the SIhigh and SI-low rats $\left(t_{(9)}=0.09 ; p=0.93\right)$, suggesting a loss of recollection in both groups. In contrast, familiarity estimates were significantly higher in the SI-high than the SI-low group $\left(t_{(9)}\right.$ $=3.5 ; p=0.007$ ) (Fig. 4I), and were also higher in the SI-high than in the young group $\left(t_{(13)}=3.06 ; p=0.009\right)$. The mean familiarity estimate was lower in the SI-low group than the young group, but this difference was not statistically significant $\left(t_{(12)}=\right.$ $1.12 ; p=0.28)$. The combined pattern of results suggests that, although both the SI-high and SI-low groups are severely impaired in recollection, the SI-high rats successfully compensate by using a familiarity-based strategy to raise their overall recognition score to a level not different from that of young rats. These observations are consistent with studies showing that some aged humans rely more heavily on familiarity in making recognition memory judgments, and that increased familiarity is associated with better performance on recognition memory tasks (Parkin and Walter, 1992; Perfect et al., 1995; Bastin and Van der Linden, 2003; Daselaar et al., 2006).

Analyses of the slope of the $z$-transformed ROC curves as a measure of ROC asymmetry provide additional evidence indicative of differences in the contribution of recollection between groups (Fig. 5A). The slopes of the group $z$-transformed ROCs were significantly different between young and aged rats (young slope, 0.68 ; aged slope, $1.11 ; F_{(1,6)}=7.17 ; p=0.04$ ), supporting the distinction in the shape of the ROC curves of young and aged animals, as well as the difference in recollection estimates between the groups. The mean slope of the individual $z$-transformed ROC was significantly less than one for young rats (asymmetric ROC; mean slope, $0.68 \pm 0.08 ; t_{(9)}=-4.2 ; p=0.002$ ), suggesting a significant contribution of recollection, but was not significantly $<1$ for aged animals (symmetrical ROC; mean slope, $0.88 \pm 0.09 ; t_{(18)}=$ $-1.3 ; p=0.23$ ), indicating that recollection did not contribute significantly to recognition performance.

Comparisons of $z$-transformed scores among groups separated based on water maze performance also confirm the ob- served differences in the amount of recollection supporting recognition performance (Fig. 5B). The slopes of the group $z$-transformed ROC functions between young, spatial-unimpaired, and spatial-impaired aged rats significantly differed (young slope, 0.68; spatialunimpaired aged group slope, 0.89; spatial-impaired aged group slope, 1.3; $\left.F_{(2,9)}=6.9 ; p=0.02\right)$. For both young rats (mean slope, $0.68 \pm 0.08 ; t_{(9)}=-4.2 ; p=$ 0.002 ) and spatial-unimpaired aged rats (mean slope, $0.75 \pm 0.07 ; t_{(7)}=-3.6 ; p=$ $0.001)$, the mean slope of the individual $z$-transformed ROCs was significantly $<1$, indicating asymmetrical ROCs and, thus, significant contributions of recollection to recognition performance. In contrast, the mean slope of the individual $z$-transformed ROC function in spatial-impaired aged rats was not significantly different from 1 (symmetrical ROC; mean slope, $\left.0.98 \pm 0.15 ; t_{(10)}=-0.11 ; p=0.92\right)$, confirming that recollection did not contribute significantly to performance.

The slopes of the $z$-transformed ROC functions in the SI-high (slope, 1.3) and SI-low group (slope, 1.05) did not significantly differ $\left(F_{(1,6)}=0.31 ; p=0.6\right)$, and the slopes of both the SI-high group (mean slope, $1.3 \pm 0.47 ; t_{(4)}=0.26 ; p=0.81$ ) and SI-low group (mean slope, $1.05 \pm 0.18 ; t_{(5)}=-1.26 ; p=0.26$ ) were not significantly different from 1 , consistent with the selective loss of recollection in aged animals that are impaired on the water maze (Fig. $5 C$ ).

\section{Analyses of hit and false alarm rates}

Analyses of the hit and false alarm rates indicate that the deficit in recollection in spatial-impaired aged rats is primarily caused by forgetting the sample stimuli. We used separate two-way ANOVAs to compare the hit and false alarm rates among groups across all bias levels and report both the overall average scores and scores for each bias level (Fig. 6). Overall, aged rats had lower average hit rates than young rats $\left(F_{(1,135)}=17.5 ; p<0.0001\right)($ Fig. $6 A)$. Post hoc comparisons showed that the hit rates of aged rats were significantly lower at the middle $\left(t_{(27)}=3.5 ; p=0.001\right)$ and lowest $\left(t_{(27)}=2.84 ; p=0.009\right)$ bias levels. Aged rats did not differ from young rats in average false alarm rates $\left(F_{(1,135)}=3.7 ; p=0.054\right)$, yet there was an interaction between groups and bias level $\left(F_{(4,135)}\right.$ $=3.01 ; p=0.02)$ such that aged rats made more false alarms than young rats at the highest bias level $\left(t_{(27)}=2.4 ; p=0.02\right)$ and fewer at the lowest bias level $\left(t_{(27)}=-2.24 ; p=0.03\right)$ (Fig. $\left.6 B\right)$.

ANOVAs comparing groups separated by performance on the water maze task also revealed that the young, spatial-unimpaired, and spatial-impaired aged rats differed in hit rates $\left(F_{(2,130)}=\right.$ 9.41; $p<0.0002)$, and showed that both the spatial unimpaired $\left(t_{(16)}=3.3 ; p=0.004\right)$ and spatial-impaired $\left(t_{(19)}=2.968 ; p=\right.$ 0.008 ) aged rats had lower hit rates than young rats (Fig. 6C). Post hoc comparisons revealed that the decrease in hits between young and spatial-impaired aged animals was significant for the highest $\left(t_{(19)}=2.32 ; p=0.03\right)$ and middle $\left(t_{(19)}=-3.04 ; p=0.007\right)$ bias levels, and was also significantly lower in spatial-unimpaired aged rats than young animals at the middle bias $\left(t_{(16)}=-3.3 ; p=\right.$ 0.005). Furthermore, only spatial-impaired aged animals made significantly fewer hits than young animals at the highest bias, which reflects the greatest contribution of recollection (Yonelinas, 2002; Eichenbaum et al., 2007). The young, spatialunimpaired, and spatial-impaired aged groups did not differ in overall false alarm rates $\left(F_{(2,130)}=2.82 ; p=0.06\right)$, nor did the 

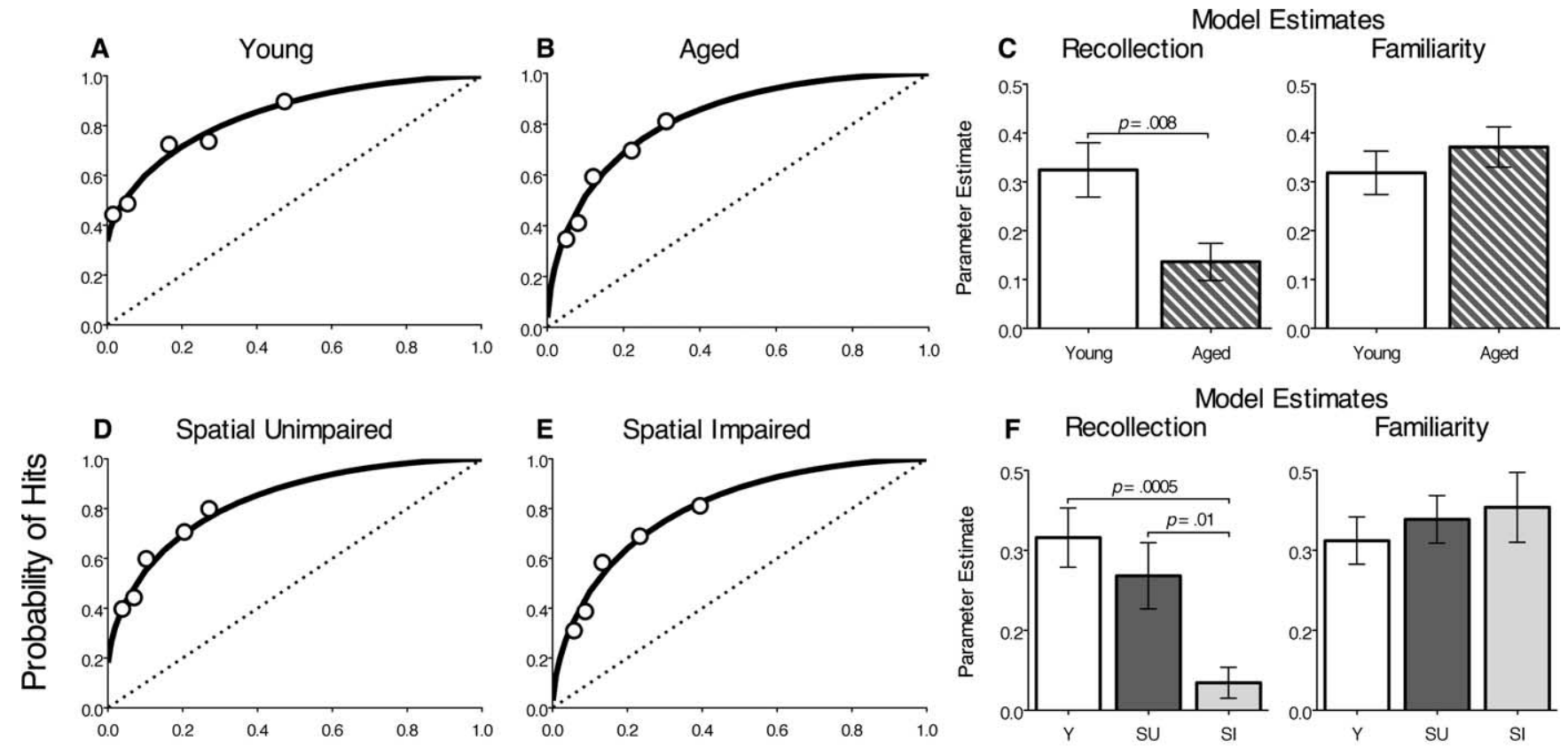

Model Estimates
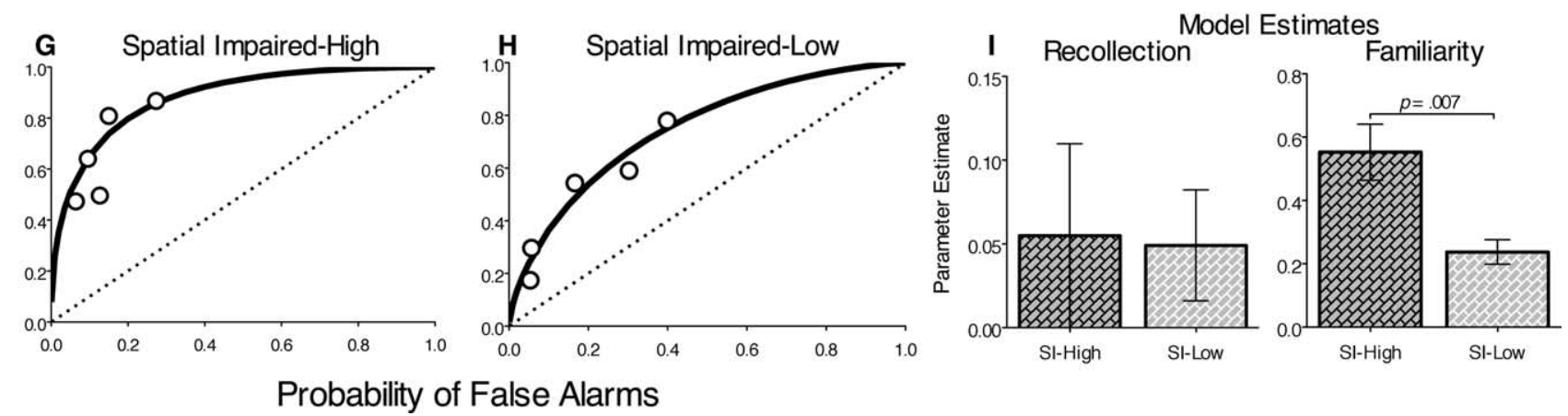

Probability of False Alarms

Figure 4. ROC functions and recollection and familiarity indices for odor recognition. Each plot shows the group mean hit and false alarm scores at each bias level (circles). Parameter estimates ( \pm SEM) of the contributions of recollection and familiarity calculated for each ROC function are shown. $A, B$, Young versus all aged rats. $C, D$, Aged rats analyzed separately for the group that was unimpaired in spatial memory (SU) and for the group that was impaired in spatial memory (SI). E, F, Spatial-impaired aged rats analyzed separately for the SI-high and SI-low subgroups.

false alarm rates differ across bias levels $\left(F_{(4,130)}=1.55 ; p=0.14\right)($ Fig. $6 D)$.

The same analyses applied to the subgroups of spatial-impaired aged rats revealed that the better performance of SIhigh rats than SI-low rats could be attributed solely to higher hit rates. SIhigh rats had a higher average hit rate than SI-low rats $\left(F_{(1,45)}=40.4 ; p<0.0001\right)$, and the post hoc analysis showed that SIhigh rats had higher hit rates on the highest $\left(t_{(9)}=6.7, p<0.0001\right)$, second highest

$\left(t_{(9)}=2.7, p=0.02\right)$, and second lowest $\left(t_{(9)}=3.0, p=0.02\right)$ bias levels (Fig. $6 E$ ). In contrast, the groups did not differ in overall false alarm rate $\left(F_{(1,45)}=3.6 ; p=0.06\right)$, nor did false alarm rates differ at any of the bias levels $\left(F_{(1,45)}=2.2 ; p=0.08\right)$ (Fig. $6 F$ ).

\section{Overall correlation between water maze performance and recognition memory}

In addition to the above group-wise comparisons, regression analyses were performed to compare the degree to which performance on the water maze could be related to the estimates of recollection and familiarity as measured by the DPSD model using the data from all groups combined. Performance on the water
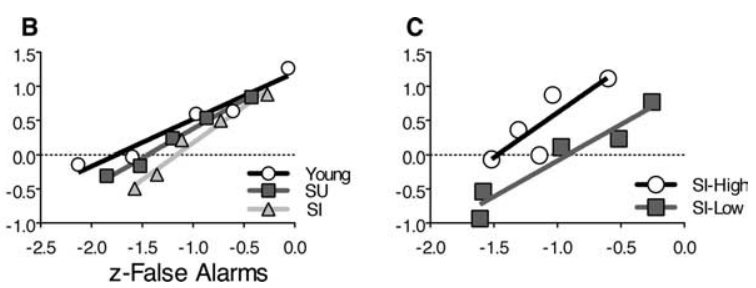

Figure 5. Best-fit linear regressions of $z$-transformed ROC functions. $\boldsymbol{A}$, Young versus all aged rats. $\boldsymbol{B}$, Aged rats analyzed separately for the group that was unimpaired in spatial memory (SU) and for the group that was impaired in spatial memory (SI). C, Spatial-impaired aged rats analyzed separately for the SI-high and SI-low subgroups.

maze was strongly related to the estimates of recollection $(\beta=$ $-0.65 ; r^{2}=0.43 ; t_{(27)}=-4.45 ; p=0.0001$ ) (Fig. $7 A$ ), consistent with substantial evidence indicating that both the water maze and the recollection component of recognition memory are dependent on the hippocampus. In contrast, there was no relationship between water maze performance and estimates of familiarity $\left(\beta=0.02 ; r^{2}=0.0001 ; t_{(27)}=0.08 ; p=0.94\right)($ Fig. $7 B)$, suggesting distinct bases of spatial memory and familiarity.

\section{Discussion}

The spatial learning ability of young and aged rats was assessed using the Morris water maze, a well-known assay of hippocampal 
A Average Hits
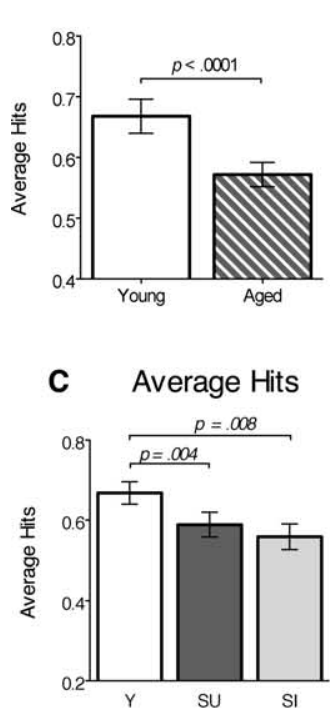

E Average Hits

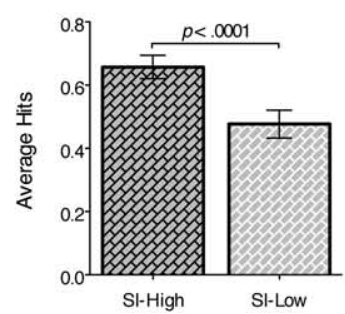

Hits

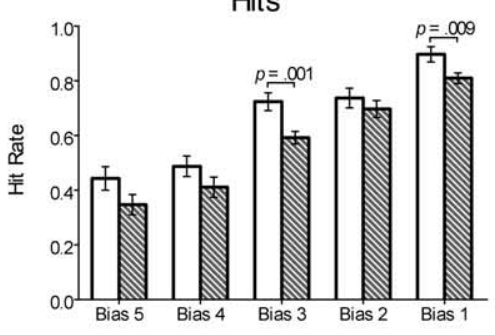

Hits

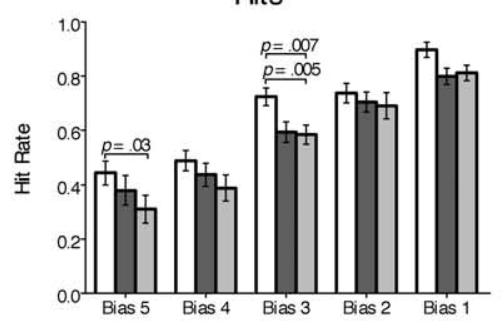

Hits

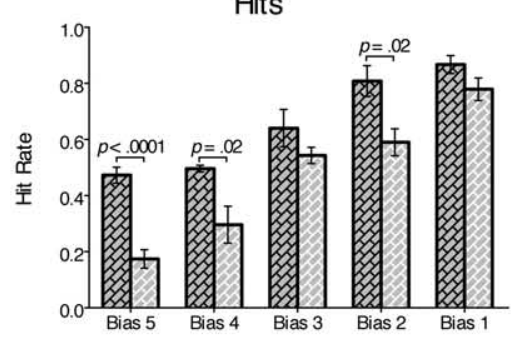

B Average False Alarms
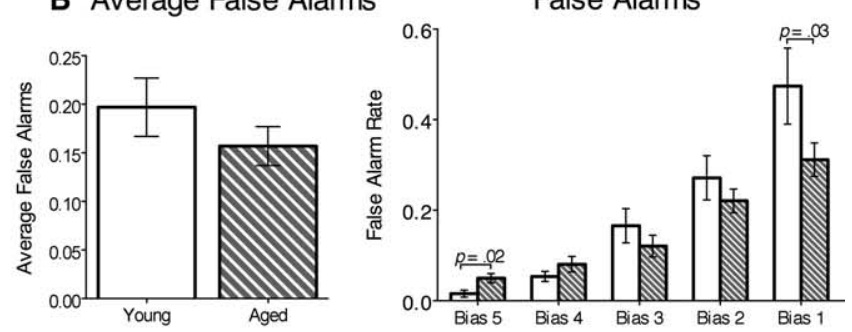

D Average False Alarms
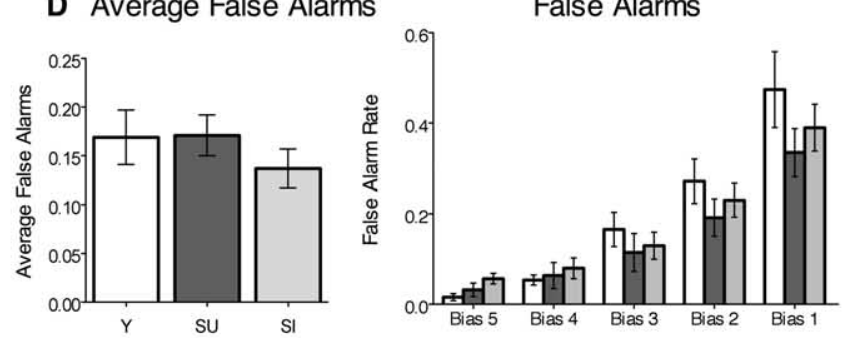

F Average False Alarms
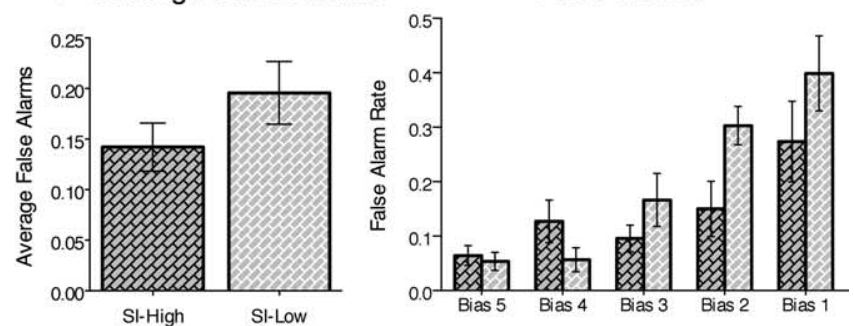

Figure 6. Group comparisons of hit and false alarm rates averaged among all bias levels, and hit and false alarm rates at each bias level (mean \pm SEM). $\boldsymbol{A}, \boldsymbol{B}$, Young versus all aged rats. $\boldsymbol{C}, \boldsymbol{D}$, Aged rats analyzed separately for the group that was unimpaired in spatial memory (SU) and for the group that was impaired in spatial memory (SI). $\boldsymbol{E}, \boldsymbol{F}$, Spatial-impaired aged rats analyzed separately for the SI-high and for the SI-low subgroups.

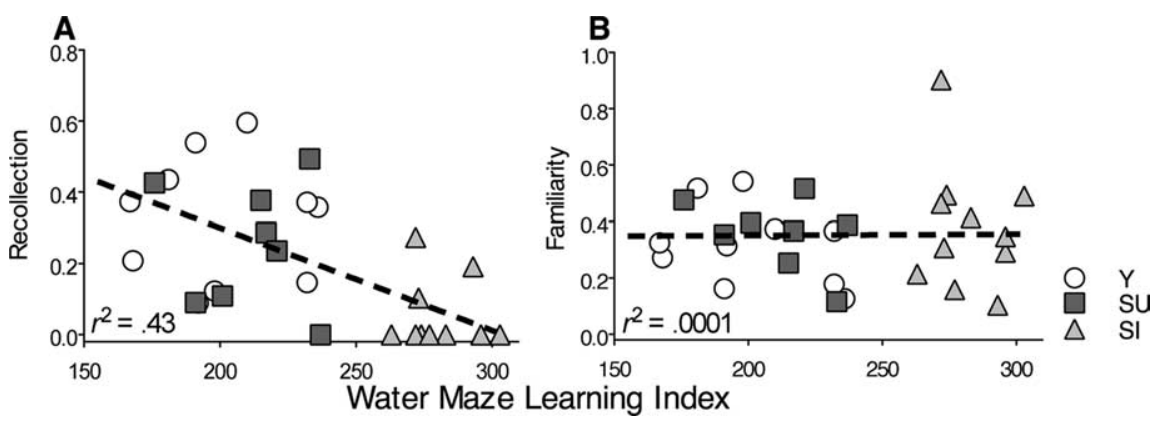

Figure 7. Correlations between spatial learning index and parameter estimates of recollection $(\boldsymbol{A})$ and familiarity $(\boldsymbol{B})$. the hippocampus in supporting episodic memory (Eichenbaum, 2000, 2004; Tulving, 2002; Rosenbaum et al., 2005).

Nonspatial recognition memory in young and aged rats was probed by assessing their ability to identify an odor as old or new across a range of response biases. Whereas overall performance (percentage correct) on the recognition memory task was not significantly reduced in the population of aged rats, signal detection analysis revealed that young and aged rats used different strategies in support of recognition performance and the extent to which they do so depends on the severity of the spatial memory impairment. The ROC curves of young rats were asymmetric and curvilinear, reflecting recognition memory supported by the contributions of both recollection and familiarity, as has been observed previously (Fortin et al., 2004). Both a recollection and a familiarity process also characterized the ROC function of aged rats that were unimpaired in water maze performance, although there was a slight decrease in recollection compared with young rats. In sharp contrast, the ROC function of aged rats that were impaired in spatial memory displayed no evidence of recollection, and instead, their ROC function was characterized solely by a familiarity process.

The recognition impairment is unlikely attributable to a def- known impairment of hippocampal function in aging and spatial memory with a more general role that has been hypothesized for function. A subset of the aged animals performed as well as young 1993). Several studies examining the neurobiological basis of this age-associated deficit in spatial learning have conclusively shown performance exhibited by spatial-impaired aged rats resembles that of rats with hippocampal lesions on various tests of learning and memory, especially tests of spatial memory (Rapp et al., 1999; Smith et al., 1999, 2000; Barnes, 2003; Gallagher et al., 2003; Wilson et al., 2006). The current experiment sought to relate the 
icit in detection or discrimination of odors, because perceptual impairments should be evident across all biases and for both recollection and familiarity, which was not observed. Furthermore, Schoenbaum et al. (2002) evaluated olfactory discrimination thresholds in rats of the same strain and from the same source and found no age-related changes.

This pattern of performance is similar to that of rats with lesions of the hippocampus (Fortin et al., 2004). These findings confirm previous observations of deficits in nonspatial nonmatching to sample tasks in rats (Zyzak et al., 1995) and monkeys (Rapp and Amaral, 1989, 1991), as well as other nonspatial learning and memory tests (Winocur, 1992; Gallagher and Rapp, 1997; Scali et al., 1997; Countryman and Gold, 2007; Pietá Dias et al., 2007). These recognition deficits are typically modest in severity, compared with the near total absence of recollection observed in the present study. Thus, the present findings serve to clarify that the previous observations of a modest deficit may reflect the combination of a severe impairment in one contribution to recognition (i.e., recollection) and relative sparing of another (i.e., familiarity). Furthermore, the present findings indicate the variability in the severity of recognition deficits is attributable to variation in the severity of the recollection deficit and the amount of compensatory increase in the contribution of familiarity among individuals. These findings also parallel data from recognition memory studies in the human cognitive aging literature, which show that normal aging results in a selective loss of recollection and relative sparing of familiarity, and the severity of the deficit is variable among individuals (Light et al., 2000; Davidson and Glisky, 2002; Bastin and Van der Linden, 2003; Howard et al., 2006).

The current data also suggest an association between hippocampal function that supports recognition memory and spatial memory. This association was evident in three of our analyses. First, performance on the water maze, a task sensitive to hippocampal function, was correlated with recognition memory performance for young and spatial-unimpaired aged animals, suggesting a common underlying memory processing function. Second, performance on the recollection component of odor recognition was more severely affected in spatial-impaired than spatial-unimpaired aged rats; the contribution of familiarity did not differ between these groups. Third, there was a strong overall correlation between water maze performance and recollection but not familiarity. Because the hippocampus is also strongly implicated in the recollection but not the familiarity component of recognition (Fortin et al., 2004), the observed relationship between odor recollection and water maze performance in aged rats also suggests a common underlying contribution of the hippocampus to normal function in both memory domains. One possibility for this common underlying function is the ability to recollect previous experiences, such that performance on probe tests in the water maze may rely on recollection of previous successful routes through the maze, much as recollection contributes to performance in the odor-recognition task.

\section{A neurobiological account of the deficit in recollection}

A mechanistic account of the related deficits in recollection and water maze performance in aged rats is suggested by findings from recording studies in aged rats exploring novel and familiar environments (Ikonen et al., 2002; Wilson et al., 2003, 2004, 2005). When exploring a novel environment after extensive exposure to another (familiar) environment, the place cells of spatial-impaired aged rats fail to form new representations of the novel environment and instead often retrieve previously stored representations of the familiar environment. These observations have been characterized as a failure in pattern separation of the contextual stimuli from the familiar and novel environments and a consequent tendency toward pattern completion of the representation of the familiar spatial context (Wilson et al., 2006).

In our odor-recognition protocol, the relevant context associated with each odor includes the preceding and following odors in the list each day, and the ability to remember the order of odors in a list depends on the hippocampus (Fortin et al., 2002). Furthermore, a recent study has shown that the hippocampus encodes a list of odor stimuli in terms of a gradually changing temporal context signal (Manns et al., 2007). It is likely that recollection of odors involves remembering these temporal contextual features to distinguish odors on the current list from previous appearances of the same odors on earlier testing sessions. The model proposed by Wilson et al. (2006), which focused on age-associated deficits in pattern separation of spatial contexts, can be extended to understand how a deficit in pattern separation might also underlie the observed impairment in odor recollection. Thus, a general age-associated reduction in pattern separation operations performed by the hippocampus could compromise the animal's ability to distinguish the temporal context of odors in the current list from that of previous appearances of the same odors in earlier lists. The result would be a catastrophic pattern of interference for the hippocampus and a consequent inability to recollect the experience of being presented with a specific odor in the current study list.

These age-associated abnormalities in pattern separation and completion may result directly from deleterious neurobiological changes in the hippocampus (Wilson et al., 2006). In particular, the impairment may be a consequence of age-associated attenuated modulation of hippocampal processing by acetylcholine (Chouinard et al., 1995; Gill and Gallagher, 1998; Sugaya et al., 1998; Nicolle et al., 1999). Models of information processing in the hippocampus suggest that acetylcholine may be important in regulating the dynamics of encoding and retrieval, such that high levels of acetylcholine bias the dynamics of the network toward encoding, whereas low levels of acetylcholine favor retrieval dynamics (Hasselmo and Wyble, 1997; Hasselmo, 2006; Giocomo and Hasselmo, 2007). Acetylcholine has also been shown to play a role in modulating interference between memories. Low levels of acetylcholine, as seen after administration of the cholinergic antagonist scopolamine, result in increased levels of interference between stored memories (De Rosa and Hasselmo, 2000), whereas higher levels of acetylcholine have been suggested to decrease levels of interference between stored activity patterns (Linster et al., 2003). Indeed, reducing cholinergic modulation experimentally reproduces in young rats the age-associated tendency of place cells to pattern complete familiar spatial representations (Ikonen et al., 2002). Also, in a study of human recognition memory, injections of scopolamine selectively modulates recollection performance but does not affect familiarity processes (Sherman et al., 2003). Thus, when injected with scopolamine, subjects show a decrease in recollection-based performance, but this performance is still qualitatively different from familiarity and normal recollection is observed after the drug has worn off. Additionally, reduced synaptic input from the entorhinal cortex that occurs selectively in aged impaired rats as defined by water maze performance, may also result in impaired pattern separation in the dentate gyrus and CA3 regions, which both contribute to pattern separation processes in the hippocampus (Wilson et al., 2006; Leutgeb et al., 2007). 


\section{References}

Albert MS (1997) The ageing brain: normal and abnormal memory. Philos Trans R Soc Lond B Biol Sci 352:1703-1709.

Barnes CA (2003) Long-term potentiation and the ageing brain. Philos Trans R Soc Lond B Biol Sci 358:765-772.

Bastin C, Van der Linden M (2003) The contribution of recollection and familiarity to recognition memory: a study of the effects of test format and aging. Neuropsychology 17:14-24.

Buckner RL (2004) Memory and executive function in aging and AD: multiple factors that cause decline and reserve factors that compensate. Neuron 44:195-208.

Chouinard ML, Gallagher M, Yasuda RP, Wolfe BB, McKinney M (1995) Hippocampal muscarinic receptor function in spatial learning-impaired aged rats. Neurobiol Aging 16:955-963.

Countryman RA, Gold PE (2007) Rapid forgetting of social transmission of food preferences in aged rats: relationship to hippocampal CREB activation. Learn Mem 14:350-358.

Craik FI, Byrd M (1982) Aging and cognitive deficits: the role of attentional resources. In: Aging and cognitive processes (Craik FI, Trehub S, eds), pp 51-110. New York: Plenum.

Crook T, Bartus RT, Ferris SH, Whitehouse P, Cohen GD, Gershon S (1986) Age-associated memory impairment: proposed diagnostic criteria and measures of clinical change. Report of a National Institute of Mental Health Work Group. Dev Neuropsychol 2:261-276.

Daselaar SM, Fleck MS, Dobbins IG, Madden DJ, Cabeza R (2006) Effects of healthy aging on hippocampal and rhinal memory functions: an eventrelated fMRI study. Cereb Cortex 16:1771-1782.

Davidson PS, Glisky EL (2002) Neuropsychological correlates of recollection and familiarity in normal aging. Cogn Affect Behav Neurosci 2:174-186

De Rosa E, Hasselmo ME (2000) Muscarinic cholinergic neuromodulation reduces proactive interference between stored odor memories during associative learning in rats. Behav Neurosci 114:32-41.

Duverne S, Habibi A, Rugg MD (2007) Regional specificity of age effects on the neural correlates of episodic retrieval. Neurobiol Aging, in press.

Eichenbaum H (2000) A cortical-hippocampal system for declarative memory. Nat Rev Neurosci 1:41-50.

Eichenbaum H (2004) Hippocampus: cognitive processes and neural representations that underlie declarative memory. Neuron 44:109-120.

Eichenbaum H, Yonelinas AP, Ranganath C (2007) The medial temporal lobe and recognition memory. Annu Rev Neurosci 30:123-152.

Fortin NJ, Agster KL, Eichenbaum HB (2002) Critical role of the hippocampus in memory for sequences of events. Nat Neurosci 5:458-462.

Fortin NJ, Wright SP, Eichenbaum H (2004) Recollection-like memory retrieval in rats is dependent on the hippocampus. Nature 431:188-191.

Gallagher M, Rapp PR (1997) The use of animal models to study the effects of aging on cognition. Annu Rev Psychol 48:339-370.

Gallagher M, Burwell R, Burchinal M (1993) Severity of spatial learning impairment in aging: development of a learning index for performance in the Morris water maze. Behav Neurosci 107:618-626.

Gallagher M, Bizon JL, Hoyt EC, Helm KA, Lund PK (2003) Effects of aging on the hippocampal formation in a naturally occurring animal model of mild cognitive impairment. Exp Gerontol 38:71-77.

Gill TM, Gallagher M (1998) Evaluation of muscarinic M2 receptor sites in basal forebrain and brainstem cholinergic systems of behaviorally characterized young and aged Long-Evans rats. Neurobiol Aging 19:217-225.

Giocomo LM, Hasselmo ME (2007) Neuromodulation by glutamate and acetylcholine can change circuit dynamics by regulating the relative influence of afferent input and excitatory feedback. Mol Neurobiol 36:184-200.

Hasselmo ME (2006) The role of acetylcholine in learning and memory. Curr Opin Neurobiol 16:710-715.

Hasselmo ME, Wyble BP (1997) Free recall and recognition in a network model of the hippocampus: simulating effects of scopolamine on human memory function. Behav Brain Res 89:1-34.

Hedden T, Gabrieli JD (2004) Insights into the ageing mind: a view from cognitive neuroscience. Nat Rev Neurosci 5:87-96.

Howard MW, Bessette-Symons B, Zhang Y, Hoyer WJ (2006) Aging selectively impairs recollection in recognition memory for pictures: evidence from modeling and receiver operating characteristic curves. Psychol Aging 21:96-106.

Ikonen S, McMahan R, Gallagher M, Eichenbaum H, Tanila H (2002) Cho- linergic system regulation of spatial representation by the hippocampus Hippocampus 12:386-397.

Jennings JM, Jacoby LL (1997) An opposition procedure for detecting agerelated deficits in recollection: telling effects of repetition. Psychol Aging 12:352-361.

Leutgeb JK, Leutgeb S, Moser MB, Moser EI (2007) Pattern separation in the dentate gyrus and CA3 of the hippocampus. Science 315:961-966.

Light LL, Prull MW, Kennison RF (2000) Divided attention, aging, and priming in exemplar generation and category verification. Mem Cognit 28:856-872.

Linster C, Maloney M, Patil M, Hasselmo ME (2003) Enhanced cholinergic suppression of previously strengthened synapses enables the formation of self-organized representations in olfactory cortex. Neurobiol Learn Mem 80:302-314.

Macmillan NA, Creelman CD (2005) Detection theory: a user's guide, Ed 2. Mahwah, NJ: Lawrence Erlbaum.

Manns JR, Howard MW, Eichenbaum H (2007) Gradual changes in hippocampal activity support remembering the order of events. Neuron 56:530-540.

Nicolle MM, Colombo PJ, Gallagher M, McKinney M (1999) Metabotropic glutamate receptor-mediated hippocampal phosphoinositide turnover is blunted in spatial learning-impaired aged rats. J Neurosci 19:9604-9610.

Parkin AJ, Walter BM (1992) Recollective experience, normal aging, and frontal dysfunction. Psychol Aging 7:290-298.

Perfect TJ, Williams RB, Anderton-Brown C (1995) Age differences in reported recollective experience are due to encoding effects, not response bias. Memory 3:169-186.

Pietá Dias C, Martins de Lima MN, Presti-Torres J, Dornelles A, Garcia VA, Siciliani Scalco F, Rewsaat Guimarães M, Constantino L, Budni P, DalPizzol F, Schröder N (2007) Memantine reduces oxidative damage and enhances long-term recognition memory in aged rats. Neuroscience 146:1719-1725.

Prull MW, Dawes LL, Martin AM 3rd, Rosenberg HF, Light LL (2006) Recollection and familiarity in recognition memory: adult age differences and neuropsychological test correlates. Psychol Aging 21:107-118.

Quamme JR, Yonelinas AP, Widaman KF, Kroll NE, Sauvé MJ (2004) Recall and recognition in mild hypoxia: using covariance structural modeling to test competing theories of explicit memory. Neuropsychologia 42:672-691.

Rapp PR, Amaral DG (1989) Evidence for task-dependent memory dysfunction in the aged monkey. J Neurosci 9:3568-3576.

Rapp PR, Amaral DG (1991) Recognition memory deficits in a subpopulation of aged monkeys resemble the effects of medial temporal lobe damage. Neurobiol Aging 12:481-486.

Rapp PR, Rosenberg RA, Gallagher M (1987) An evaluation of spatial information processing in aged rats. Behav Neurosci 101:3-12.

Rapp PR, Stack EC, Gallagher M (1999) Morphometric studies of the aged hippocampus: I. Volumetric analysis in behaviorally characterized rats. J Comp Neurol 403:459-470.

Robitsek R, Guttentag L, Fortin N, Eichenbaum H (2006) Recognition memory in rodent normal aging. Soc Neurosci Abstr 32:66.18.

Rosenbaum RS, Köhler S, Schacter DL, Moscovitch M, Westmacott R, Black SE, Gao F, Tulving E (2005) The case of K.C.: contributions of a memory-impaired person to memory theory. Neuropsychologia 43:989-1021.

Rosenzweig ES, Barnes CA (2003) Impact of aging on hippocampal function: plasticity, network dynamics, and cognition. Prog Neurobiol 69:143-179.

Scali C, Giovannini MG, Bartolini L, Prosperi C, Hinz V, Schmidt B, Pepeu G (1997) Effect of metrifonate on extracellular brain acetylcholine and object recognition in aged rats. Eur J Pharmacol 325:173-180.

Schoenbaum G, Nugent S, Saddoris MP, Gallagher M (2002) Teaching old rats new tricks: age-related impairments in olfactory reversal learning. Neurobiol Aging 23:555-564.

Sherman SJ, Atri A, Hasselmo ME, Stern CE, Howard MW (2003) Scopolamine impairs human recognition memory: data and modeling. Behav Neurosci 117:526-539.

Smith TD, Calhoun ME, Rapp PR (1999) Circuit and morphological specificity of synaptic change in the aged hippocampal formation. Neurobiol Aging 20:357-358, 1999; discussion 359-360.

Smith TD, Adams MM, Gallagher M, Morrison JH, Rapp PR (2000) Circuit-specific alterations in hippocampal synaptophysin immunoreac- 
tivity predict spatial learning impairment in aged rats. J Neurosci 20:6587-6593.

Sugaya K, Greene R, Personett D, Robbins M, Kent C, Bryan D, Skiba E, Gallagher M, McKinney M (1998) Septo-hippocampal cholinergic and neurotrophin markers in age-induced cognitive decline. Neurobiol Aging 19:351-361.

Toth JP, Parks CM (2006) Effects of age on estimated familiarity in the process dissociation procedure: the role of noncriterial recollection. Mem Cognit 34:527-537.

Tulving E (2002) Episodic memory: from mind to brain. Annu Rev Psychol 53:1-25.

Wilson IA, Ikonen S, McMahan RW, Gallagher M, Eichenbaum H, Tanila H (2003) Place cell rigidity correlates with impaired spatial learning in aged rats. Neurobiol Aging 24:297-305.

Wilson IA, Ikonen S, Gureviciene I, McMahan RW, Gallagher M, Eichenbaum $\mathrm{H}$, Tanila $\mathrm{H}$ (2004) Cognitive aging and the hippocampus: how old rats represent new environments. J Neurosci 24:3870-3878.

Wilson IA, Ikonen S, Gallagher M, Eichenbaum H, Tanila H (2005) Ageassociated alterations of hippocampal place cells are subregion specific. J Neurosci 25:6877-6886.
Wilson IA, Gallagher M, Eichenbaum H, Tanila H (2006) Neurocognitive aging: prior memories hinder new hippocampal encoding. Trends Neurosci 29:662-670.

Winocur G (1992) A comparison of normal old rats and young adult rats with lesions to the hippocampus or prefrontal cortex on a test of matching-to-sample. Neuropsychologia 30:769-781.

Wixted JT (2007) Dual-process theory and signal-detection theory of recognition memory. Psychological Rev 114:152-176.

Yonelinas AP (2002) The nature of recollection and familiarity: a review of 30 years of research. J Mem Lang 48:441-517.

Yonelinas AP, Parks CM (2007) Receiver operating characteristics (ROCs) in recognition memory: a review. Psychol Bull 133:800-832.

Yonelinas AP, Kroll NE, Dobbins I, Lazzara M, Knight RT (1998) Recollection and familiarity deficits in amnesia: convergence of remember-know, process dissociation, and receiver operating characteristic data. Neuropsychology 12:323-339.

Zyzak DR, Otto T, Eichenbaum H, Gallagher M (1995) Cognitive decline associated with normal aging in rats: a neuropsychological approach. Learn Mem 2:1-16. 